\title{
On the vector-valued Fourier transform and compatibility of operators
}

\author{
by
}

\author{
In SOOK PARK (Taejeon)
}

\begin{abstract}
Let $\mathbb{G}$ be a locally compact abelian group and let $1<p \leq 2$. $\mathbb{G}^{\prime}$ is the dual group of $\mathbb{G}$, and $p^{\prime}$ the conjugate exponent of $p$. An operator $T$ between Banach spaces $X$ and $Y$ is said to be compatible with the Fourier transform $F^{\mathbb{G}}$ if $F^{\mathbb{G}} \otimes T: L_{p}(\mathbb{G}) \otimes X \rightarrow$ $L_{p^{\prime}}\left(\mathbb{G}^{\prime}\right) \otimes Y$ admits a continuous extension $\left[F^{\mathbb{G}}, T\right]:\left[L_{p}(\mathbb{G}), X\right] \rightarrow\left[L_{p^{\prime}}\left(\mathbb{G}^{\prime}\right), Y\right]$. Let $\mathcal{F} \mathcal{T}_{p}^{\mathbb{G}}$ denote the collection of such $T$ 's. We show that $\mathcal{F} \mathcal{T}_{p}^{\mathbb{R}} \times \mathbb{G}=\mathcal{F} \mathcal{T}_{p}^{\mathbb{Z}} \times \mathbb{G}=\mathcal{F} \mathcal{T}_{p}^{n} \times \mathbb{G}$ for any $\mathbb{G}$ and positive integer $n$. Moreover, if the factor group of $\mathbb{G}$ by its identity component is a direct sum of a torsion-free group and a finite group with discrete topology then $\mathcal{F} \mathcal{T}_{p}^{\mathbb{G}}=\mathcal{F} \mathcal{T}_{p}^{\mathbb{Z}}$
\end{abstract}

1. Introduction. A locally compact abelian group means a topological abelian group whose topology is locally compact Hausdorff and which is equipped with a Haar measure. The real line $\mathbb{R}$, the discrete group of integers $\mathbb{Z}$ and the circle group $\mathbb{T}$ are important examples. Further information can be found in [3], [5] and [13]. Let $\mathbb{G}$ be a locally compact abelian group and $\mathbb{G}^{\prime}$ its dual (character) group. Then the Haar measure of $\mathbb{G}^{\prime}$ can be determined so that Parseval's identity holds with constant 1 . For $1 \leq r<\infty$, we denote by $\left[L_{r}\left(\mathbb{G}, \mu_{\mathbb{G}}\right), X\right]$ the Banach space of all $\mu_{\mathbb{G}}$-measurable functions $\mathbf{f}: \mathbb{G} \rightarrow X$ such that $\left\|\mathbf{f} \mid L_{r}(\mathbb{G})\right\|:=\left(\int_{\mathbb{G}}\|\mathbf{f}(s)\|^{r} d \mu_{\mathbb{G}}(s)\right)^{1 / r}$ is finite.

Let $\mathbb{G}$ be a fixed infinite locally compact abelian group and $1<p \leq 2$. Then $F^{\mathbb{G}}$ denotes the Fourier transform from $L_{p}(\mathbb{G})$ into $L_{p^{\prime}}\left(\mathbb{G}^{\prime}\right)$. For a bounded linear operator $T$ between Banach spaces $X$ and $Y$,

$$
F^{\mathbb{G}} \otimes T: \sum_{k=1}^{n} f_{k} \otimes \mathbf{x}_{k} \mapsto \sum_{k=1}^{n} F^{\mathbb{G}} f_{k} \otimes T \mathbf{x}_{k}
$$

yields a well defined map from $L_{p}(\mathbb{G}) \otimes X$ into $L_{p^{\prime}}\left(\mathbb{G}^{\prime}\right) \otimes Y$. The operator $T$ is said to be compatible with $F^{\mathbb{G}}$, or have $\mathbb{G}$-Fourier type $p$, if the operator

2000 Mathematics Subject Classification: Primary 47L20; Secondary 43A25.

Key words and phrases: Banach space, operator, Fourier transform, vector-valued function, locally compact abelian group, dual group.

This work is supported by BK21 project. 
$F^{\mathbb{G}} \otimes T: L_{p}(\mathbb{G}) \otimes X \rightarrow L_{p^{\prime}}\left(\mathbb{G}^{\prime}\right) \otimes Y$ admits a continuous extension

$$
\left[F^{\mathbb{G}}, T\right]:\left[L_{p}(\mathbb{G}), X\right] \rightarrow\left[L_{p^{\prime}}\left(\mathbb{G}^{\prime}\right), Y\right]
$$

For such $T$, we let

$$
\left\|T \mid \mathcal{F} \mathcal{T}_{p}^{\mathbb{G}}\right\|:=\left\|\left[F^{\mathbb{G}}, T\right]:\left[L_{p}(\mathbb{G}), X\right] \rightarrow\left[L_{p^{\prime}}\left(\mathbb{G}^{\prime}\right), Y\right]\right\| .
$$

The norm defined above is invariant under changing the Haar measure of $\mathbb{G}$. The class of these operators is a Banach ideal, denoted by $\mathcal{F} \mathcal{T}_{p}^{\mathbb{G}}$. The definition and notation follow those of [11].

It is known that $\mathcal{F} \mathcal{T}_{p}^{\mathbb{R}}=\mathcal{F} \mathcal{T}_{p}^{\mathbb{Z}}=\mathcal{F} \mathcal{T}_{p}^{\mathbb{T}}$ (see [11]), but the problem whether the operator ideal $\mathcal{F} \mathcal{T}_{p}^{\mathbb{G}}$ depends on $\mathbb{G}$ or not is unsolved. There are several results about $\mathrm{FT}_{p}^{\mathbb{G}}$, the class of Banach spaces whose identity operators are compatible with $F^{\mathbb{G}}$. These are immediately extended to the case of $\mathcal{F} \mathcal{T}_{p}^{\mathbb{G}}$ by replacing the identity operator on a Banach space $X$ with $T$. Peetre [10] who introduced the concept of Banach space of Fourier type $p$ proved that $X$ belongs to $\mathrm{FT}_{p}^{\mathbb{R}}$ if and only if the dual space $X^{\prime}$ belongs to $\mathrm{FT}_{p}^{\mathbb{R}}$. In fact, $T$ belongs to $\mathcal{F} \mathcal{T}_{p}^{\mathbb{G}}$ if and only if the dual operator $T^{\prime}$ belongs to $\mathcal{F} \mathcal{T}_{p}^{\mathbb{G}^{\prime}}$, i.e. $\left\|T\left|\mathcal{F} \mathcal{T}_{p}^{\mathbb{G}}\|=\| T^{\prime}\right| \mathcal{F} \mathcal{T}_{p}^{\mathbb{G}^{\prime}}\right\|$ for any locally compact abelian group $\mathbb{G}$. Bourgain [2] showed that $\mathrm{FT}_{p}^{\mathbb{T}} \subset \mathrm{FT}_{p}^{\mathbb{R}}$ and König [8] modified Kwapień's argument [9] to show that $\mathrm{FT}_{p}^{\mathbb{R}}=\mathrm{FT}_{p}^{\mathbb{T}}$ and extended this to $\mathrm{FT}_{p}^{\mathbb{G}}=\mathrm{FT}_{p}^{\mathbb{T}}$ if $\mathbb{G}$ is one of $\mathbb{R}^{m}$ and $\mathbb{T}^{m}$, where $m$ is a positive integer. García-Cuerva, Kazarian and Torrea [4] and Andersson [1] showed independently that $\mathrm{FT}_{p}^{\mathbb{G}}=\mathrm{FT}_{p}^{\mathbb{Z}}$ whenever $\mathbb{G}$ is one of $\mathbb{T}^{m}, \mathbb{T}^{\infty}, \mathbb{R}^{m}, \mathbb{Z}^{m}$ and $\mathbb{Z}^{\infty}$. Andersson [1] also proved that $\left\|I_{X}\left|\mathcal{F} \mathcal{T}_{p}^{\mathbb{H}}\|\leq\| I_{X}\right| \mathcal{F} \mathcal{T}_{p}^{\mathbb{G}}\right\|$ when $\mathbb{H}$ is an open subgroup of $\mathbb{G}$, and that $\mathrm{FT}_{p}^{\mathbb{E}}=\mathrm{FT}_{p}^{\mathbb{Z}}$ if $\mathbb{E}$ is a nontrivial torsion free abelian group with the discrete topology.

In this paper we characterize $\mathcal{F} \mathcal{T}_{p}^{\mathbb{G}}$ partly as follows. In Section 2, we show for every locally compact abelian group $\mathbb{G}$ that $\left\|T \mid \mathcal{F} \mathcal{T}_{p}^{\mathbb{R} \times \mathbb{G}}\right\|$ is equivalent to $\left\|T \mid \mathcal{F} \mathcal{T}_{p}^{\mathbb{Z} \times \mathbb{G}}\right\|$, and $\left\|T\left|\mathcal{F} \mathcal{T}_{p}^{\mathbb{Z}^{n} \times \mathbb{G}}\|=\| T\right| \mathcal{F} \mathcal{T}_{p}^{\mathbb{Z} \times \mathbb{G}}\right\|$. The cartesian product means the direct product. By applying these, we easily obtain some results in the above paragraph and the relation $\mathcal{F} \mathcal{T}_{p}^{k} \times \mathbb{Z}^{l} \times \mathbb{T}^{m} \times \mathbb{G}=\mathcal{F} \mathcal{T}_{p}^{\mathbb{Z} \times \mathbb{G}}$ for any nonnegative integers $k, l, m$ with $k+l+m \geq 1$. In Section 3 , we combine the results of Section 2 with the properties of locally compact abelian groups to show that $\mathcal{F} \mathcal{T}_{p}^{\mathbb{R}^{k} \times \mathbb{Z}^{l} \times \mathbb{F}}=\mathcal{F} \mathcal{T}_{p}^{\mathbb{Z}}$ for any compact abelian group $\mathbb{F}$ with finitely many components. Moreover, we show that if $\mathbb{G} \cong$ $\mathbb{R}^{k} \times[$ torsion-free group with discrete topology $] \times[$ compact group with finitely many components] then $\mathcal{F} \mathcal{T}_{p}^{\mathbb{G}}=\mathcal{F} \mathcal{T}_{p}^{\mathbb{Z}}$. If the factor group of a locally compact abelian group $\mathbb{G}$ by the identity component is in the form of [torsion-free group] $\times\left[\right.$ finite group] with the discrete topology, then we have $\mathcal{F} \mathcal{T}_{p}^{\mathbb{G}}=\mathcal{F T}_{p}^{\mathbb{Z}}$.

From now on, $X$ and $Y$ are Banach spaces and $T: X \rightarrow Y$ is a bounded linear operator. We denote the dual group of $\mathbb{G}$ by $\mathbb{G}^{\prime}$. We use the fact 
that $\mathbb{R}^{\prime}=\mathbb{R}$ and $\mathbb{Z}^{\prime}=\mathbb{T}$. We use the abbreviation LCA for "locally compact abelian". The term "isomorphic" means "topologically and algebraically isomorphic". The integral of a vector-valued function is the Bochner integral.

2. Classifying $\mathcal{F} \mathcal{T}_{p}^{\mathbb{G}}$ via direct product. First we observe that the proof in [4] of the fact $\mathrm{FT}_{p}^{\mathbb{R}}=\mathrm{FT}_{p}^{\mathbb{Z}}$ can be modified to yield the following:

Proposition 1. For any LCA group $\mathbb{G}$, we have the inequalities

$$
\left\|T\left|\mathcal{F} \mathcal{T}_{p}^{\mathbb{R} \times \mathbb{G}}\|\leq\| T\right| \mathcal{F} \mathcal{T}_{p}^{\mathbb{Z} \times \mathbb{G}}\right\| \leq \frac{\pi}{2}\left\|T \mid \mathcal{F} \mathcal{T}_{p}^{\mathbb{R} \times \mathbb{G}}\right\|,
$$

and hence $\mathcal{F} \mathcal{T}_{p}^{\mathbb{R} \times \mathbb{G}}=\mathcal{F} \mathcal{T}_{p}^{\mathbb{Z} \times \mathbb{G}}$.

Proof. For arbitrary $\delta>0$, let

$$
\mathbf{f}(s, t)=\sum_{m} \chi_{[\delta(m-1 / 2), \delta(m+1 / 2)]}(s) \mathbf{g}_{m}(t),
$$

where the summation is over $\mathbb{Z}, \mathbf{g}_{m}$ is an $X$-valued simple function on $\mathbb{G}$ and $\mathbf{g}_{m}=0$ except for finitely many $m$. Note that the set of all $X$-valued functions $\mathbf{f}$ as above is dense in $\left[L_{p}(\mathbb{R} \times \mathbb{G}), X\right]$. We compute

$$
\begin{aligned}
\left\|\mathbf{f} \mid L_{p}(\mathbb{R} \times \mathbb{G})\right\| & =\left(\int_{\mathbb{R} \times \mathbb{G}}\left\|\sum_{m} \chi_{[\delta(m-1 / 2), \delta(m+1 / 2)]}(s) \mathbf{g}_{m}(t)\right\|^{p} d s d t\right)^{1 / p} \\
& =\delta^{1 / p}\left(\int_{\mathbb{G}} \sum_{m}\left\|\mathbf{g}_{m}(t)\right\|^{p} d t\right)^{1 / p} .
\end{aligned}
$$

Since $\int_{\delta(m-1 / 2)}^{\delta(m+1 / 2)} \exp (i \widetilde{s} s) d s=\exp (i m \delta \widetilde{s}) \frac{\sin (\delta \widetilde{s} / 2)}{\widetilde{s} / 2}$, we have

Hence

$$
\begin{aligned}
\widehat{T \mathbf{f}}(\widetilde{s}, \widetilde{t}) & =\iint_{\mathbb{G}} \sum_{\mathbb{R}} T \mathbf{g}_{m}(t) \chi_{[\delta(m-1 / 2), \delta(m+1 / 2)]}(s) \exp (i \widetilde{s} s)(t, \widetilde{t}) d s d t \\
& =\int_{\mathbb{G}} \sum_{m} T \mathbf{g}_{m}(t)(t, \widetilde{t}) \exp (i \delta m \widetilde{s}) \frac{\sin (\delta \widetilde{s} / 2)}{\widetilde{s} / 2} d t .
\end{aligned}
$$

$$
\begin{aligned}
\left\|\left[F^{\mathbb{R} \times \mathbb{G}} T\right] \mathbf{f}\right\| \|^{p^{\prime}} & \\
= & \int_{\mathbb{G}^{\prime}} \int_{\mathbb{R}}\left\|\int_{\mathbb{G}} \sum_{m} T \mathbf{g}_{m}(t)(t, \widetilde{t}) \exp (i \delta m \widetilde{s}) \frac{\sin (\delta \widetilde{s} / 2)}{\widetilde{s} / 2} d t\right\|^{p^{\prime}} \frac{1}{2 \pi} d \widetilde{s} d \widetilde{t} \\
= & \delta^{p^{\prime}} \int_{\mathbb{G}^{\prime}} \int_{\mathbb{R}}\left\|\int_{\mathbb{G}} \sum_{m} T \mathbf{g}_{m}(t)(t, \widetilde{t}) \exp (i \delta m \widetilde{s}) \frac{\sin (\delta \widetilde{s} / 2)}{\delta \widetilde{s} / 2} d t\right\|^{p^{\prime}} \frac{1}{2 \pi} d \widetilde{s} d \widetilde{t} \\
= & \left.\delta^{p^{\prime}-1} \int_{\mathbb{G}^{\prime}} \int_{\mathbb{R}} \frac{\sin (\widetilde{s} / 2)}{\widetilde{s} / 2}\right|^{p^{\prime}} \\
& \times\left\|\int_{\mathbb{G}} \sum_{k}\left(\sum_{m} T \mathbf{g}_{m}(t) \chi_{\{m\}}(k)\right)(t, \widetilde{t}) \exp (i k \widetilde{s}) d t\right\|^{p^{\prime}} \frac{1}{2 \pi} d \widetilde{s} d \widetilde{t}
\end{aligned}
$$




$$
\begin{aligned}
& =\delta^{p^{\prime}-1} \int_{\mathbb{G}^{\prime}} \int_{-\pi}^{\pi} \sum_{n}\left|\frac{\sin (\widetilde{s} / 2)}{\widetilde{s} / 2-n \pi}\right|^{p^{\prime}}\left\|\left[F^{\mathbb{Z} \times \mathbb{G}}, T\right]\left(\sum_{m} \mathbf{g}_{m} \chi_{\{m\}}\right)\right\|^{p^{\prime}} \frac{1}{2 \pi} d \widetilde{s} d \widetilde{t} \\
& \leq \delta^{p^{\prime}-1}\left\|T \mid \mathcal{F} \mathcal{T}_{p}^{\mathbb{Z} \times \mathbb{G}}\right\|^{p^{\prime}}\left(\int_{\mathbb{G}} \sum_{m}\left\|\mathbf{g}_{m}(t)\right\|^{p} d t\right)^{p^{\prime} / p} \\
& =\left\|T\left|\mathcal{F} \mathcal{T}_{p}^{\mathbb{Z} \times \mathbb{G}}\left\|^{p^{\prime}}\right\| \mathbf{f}\right| L_{p}(\mathbb{R} \times \mathbb{G})\right\|^{p^{\prime}} \quad \text { by }(1),
\end{aligned}
$$

where we have used the inequality $\sum_{n}\left|\frac{\sin \widetilde{s}}{\widetilde{s}-n \pi}\right|^{p^{\prime}} \leq 1$ for any real $\widetilde{s} \neq n \pi$ and $2 \leq p^{\prime}<\infty$ (see [7])

Therefore we have $\left\|T\left|\mathcal{F} \mathcal{T}_{p}^{\mathbb{R} \times \mathbb{G}}\|\leq\| T\right| \mathcal{F} \mathcal{T}_{p}^{\mathbb{Z} \times \mathbb{G}}\right\|$ and $\mathcal{F} \mathcal{T}_{p}^{\mathbb{Z} \times \mathbb{G}} \subseteq \mathcal{F} \mathcal{T}_{p}^{\mathbb{R} \times \mathbb{G}}$.

For the right inequality of the proposition let $\mathbf{f}(k, t)=\sum_{m} \mathbf{g}_{m}(t) \chi_{\{m\}}(k)$, where $\mathbf{g}_{m}$ is an $X$-valued simple function and $\mathbf{g}_{m}=0$ except for finitely many $m$. By a density argument it is enough to consider $\mathbf{f}$ of the above form. Now we have

$$
\begin{aligned}
\left\|\mathbf{f} \mid L_{p}(\mathbb{Z} \times \mathbb{G})\right\| & =\left(\int_{\mathbb{G}} \sum_{k}\left\|\sum_{m} \mathbf{g}_{m}(t) \chi_{\{m\}}(k)\right\|^{p} d t\right)^{1 / p} \\
& =\left(\int \sum_{\mathbb{G}}\left\|\mathbf{g}_{m}(t)\right\|^{p} d t\right)^{1 / p} ;
\end{aligned}
$$

and

$$
\begin{aligned}
\widehat{T \mathbf{f}}(\widetilde{s}, \widetilde{t}) & =\int_{\mathbb{G}} \sum_{k}\left(\sum_{m} T \mathbf{g}_{m}(t) \chi_{\{m\}}(k)\right) \exp (i \widetilde{s} k)(t, \widetilde{t}) d t \\
& =\int_{\mathbb{G}} \sum_{m} T \mathbf{g}_{m}(t) \exp (i \widetilde{s} m)(t, \widetilde{t}) d t .
\end{aligned}
$$

We use the identity

$$
\exp (i m \widetilde{s})=\int_{\mathbb{R}} \frac{\widetilde{s} / 2}{\sin (\widetilde{s} / 2)} \chi_{[m-1 / 2, m+1 / 2]}(s) \exp (i s \widetilde{s}) d s
$$

to obtain the following inequality (we abbreviate $\chi_{[m-1 / 2, m+1 / 2]}$ to $\chi_{m}$ ):

$$
\begin{aligned}
& \left\|\left[F^{\mathbb{Z} \times \mathbb{G}}, T\right] \mathbf{f}\right\|^{p^{\prime}} \\
& =\frac{1}{2 \pi} \int_{\mathbb{G}^{\prime}} \int_{-\pi}^{\pi}\left\|\int_{\mathbb{G}} \sum_{m} T \mathbf{g}_{m}(t)(t, \widetilde{t}) \int_{\mathbb{R}} \frac{\widetilde{s} / 2}{\sin (\widetilde{s} / 2)} \chi_{m}(s) \exp (i \widetilde{s} s) d s d t\right\|^{p^{\prime}} d \widetilde{s} d \widetilde{t} \\
& \leq \frac{1}{2 \pi} \int_{\mathbb{G}^{\prime}} \int_{-\pi}^{\pi}\left|\frac{\widetilde{s} / 2}{\sin (\widetilde{s} / 2)}\right|^{p^{\prime}}\left\|\iint_{\mathbb{G}} \sum_{\mathbb{R}} T \mathbf{g}_{m}(t) \chi_{m}(s)(t, \widetilde{t}) \exp (i \widetilde{s} s) d s d t\right\|^{p^{\prime}} d \widetilde{s} d \widetilde{t} \\
& \text { (because } \left.\left|\frac{\widetilde{s} / 2}{\sin (\widetilde{s} / 2)}\right| \leq \frac{\pi}{2} \text { for }-\pi \leq \widetilde{s} \leq \pi\right)
\end{aligned}
$$




$$
\begin{aligned}
& \leq \frac{1}{2 \pi}\left(\frac{\pi}{2}\right)^{p^{\prime}} \int_{\mathbb{G}^{\prime}} \int_{\mathbb{R}}\left\|\int_{\mathbb{G}} \int_{\mathbb{R}} \sum_{m} T \mathbf{g}_{m}(t) \chi_{m}(s)(t, \widetilde{t}) \exp (i \widetilde{s} s) d s d t\right\|^{p^{\prime}} d \widetilde{s} d \widetilde{t} \\
& =\left(\frac{\pi}{2}\right)^{p^{\prime}}\left\|\left[F^{\mathbb{R} \times \mathbb{G}}, T\right]\left(\sum_{m} \mathbf{g}_{m} \chi_{m}\right)\right\|^{p^{\prime}} \\
& \leq\left(\frac{\pi}{2}\right)^{p^{\prime}}\left\|T\left|\mathcal{F} \mathcal{T}_{p}^{\mathbb{R} \times \mathbb{G}}\left\|p^{\prime}\right\| \sum_{m} \mathbf{g}_{m} \chi_{m}\right| L_{p}(\mathbb{R} \times \mathbb{G})\right\|^{p^{\prime}} \\
& \left(\operatorname{since}\left\|\sum_{m} \mathbf{g}_{m} \chi_{m} \mid L_{p}(\mathbb{R} \times \mathbb{G})\right\|=\left(\int_{\mathbb{G}} \sum_{m}\left\|\mathbf{g}_{m}(t)\right\|^{p} d t\right)^{1 / p}\right) \\
& =\left(\frac{\pi}{2}\right)^{p^{\prime}}\left\|T\left|\mathcal{F} \mathcal{T}_{p}^{\mathbb{R} \times \mathbb{G}}\left\|^{p^{\prime}}\right\| \mathbf{f}\right| L_{p}(\mathbb{Z} \times \mathbb{G})\right\|^{p^{\prime}} \quad \text { by }(2) .
\end{aligned}
$$

Therefore we have $\left\|T\left|\mathcal{F} \mathcal{T}_{p}^{\mathbb{Z} \times \mathbb{G}}\left\|\leq \frac{\pi}{2}\right\| T\right| \mathcal{F} \mathcal{T}_{p}^{\mathbb{R} \times \mathbb{G}}\right\|$ and $\mathcal{F} \mathcal{T}_{p}^{\mathbb{R} \times \mathbb{G}} \subseteq \mathcal{F} \mathcal{T}_{p}^{\mathbb{Z} \times \mathbb{G}}$.

According to Theorem 6.3 of [4], for any LCA group $\mathbb{G}$ and operator $T$, $\left\|T\left|\mathcal{F} \mathcal{T}_{p}^{\mathbb{G}}\|=\| T^{\prime}\right| \mathcal{F} \mathcal{T}_{p}^{\mathbb{G}^{\prime}}\right\|$. By applying this property, we have the following:

Proposition 2. For every LCA group $\mathbb{G}, \mathcal{F}_{p}^{\mathbb{R} \times \mathbb{G}}=\mathcal{F} \mathcal{T}_{p}^{\mathbb{T} \times \mathbb{G}}$.

Proof. By Proposition 1 we have

$$
\left\|T\left|\mathcal{F} \mathcal{T}_{p}^{\mathbb{R} \times \mathbb{G}}\|=\| T^{\prime}\right| \mathcal{F} \mathcal{T}_{p}^{\mathbb{R} \times \mathbb{G}^{\prime}}\right\| \leq\left\|T^{\prime}\left|\mathcal{F} \mathcal{T}_{p}^{\mathbb{Z} \times \mathbb{G}^{\prime}}\|=\| T\right| \mathcal{F} \mathcal{T}_{p}^{\mathbb{T} \times \mathbb{G}}\right\|
$$

Similarly

$$
\left\|T\left|\mathcal{F} \mathcal{T}_{p}^{\mathbb{T} \times \mathbb{G}}\left\|\leq \frac{\pi}{2}\right\| T\right| \mathcal{F} \mathcal{T}_{p}^{\mathbb{R} \times \mathbb{G}}\right\|
$$

From Propositions 1 and 2, we conclude that $\mathcal{F} \mathcal{T}_{p}^{\mathbb{R} \times \mathbb{G}}=\mathcal{F} \mathcal{T}_{p}^{\mathbb{Z} \times \mathbb{G}}=$ $\mathcal{F} \mathcal{T}_{p}^{\mathbb{T} \times \mathbb{G}}$ for every LCA group $\mathbb{G}$. We also have the following two corollaries.

Corollary 3. $\mathcal{F} \mathcal{T}_{p}^{\mathbb{R}^{n}}=\mathcal{F} \mathcal{T}_{p}^{\mathbb{Z}^{n}}=\mathcal{F} \mathcal{T}_{p}^{\mathbb{T}^{n}}$ for every integer $n \geq 1$

Proof. By Proposition 1 we have

$$
\mathcal{F} \mathcal{T}_{p}^{\mathbb{R}^{n}}=\mathcal{F} \mathcal{T}_{p}^{\mathbb{R} \times \mathbb{R}^{n-1}}=\mathcal{F} \mathcal{T}_{p}^{\mathbb{Z} \times \mathbb{R}^{n-1}}
$$

We continue this to obtain



Similarly we apply Proposition $2 n$ times to obtain

$$
\mathcal{F} \mathcal{T}_{p}^{\mathbb{R}^{n}}=\mathcal{F} \mathcal{T}_{p}^{\mathbb{T}^{n}}
$$

COROLlary 4. $\mathcal{F} \mathcal{T}_{p}^{\mathbb{R}^{n} \times \mathbb{G}}=\mathcal{F} \mathcal{T}_{p}^{\mathbb{Z}^{n} \times \mathbb{G}}=\mathcal{F} \mathcal{T}_{p}^{\mathbb{T}^{n} \times \mathbb{G}}$ for every LCA group $\mathbb{G}$.

Proof. The proof is similar to that of Corollary 3.

LEMMA 5. For every LCA group $\mathbb{G}$ and every positive integer $n$,

$$
\left\|T\left|\mathcal{F} \mathcal{T}_{p}^{\mathbb{Z}^{n} \times \mathbb{G}}\|=\| T\right| \mathcal{F} \mathcal{T}_{p}^{\mathbb{Z} \times \mathbb{G}}\right\|
$$

and therefore $\mathcal{F} \mathcal{T}_{p}^{\mathbb{Z}^{n} \times \mathbb{G}}=\mathcal{F} \mathcal{T}_{p}^{\mathbb{Z} \times \mathbb{G}}$. 
Proof. It is enough to prove this statement for $n=2$. Proposition 1.2 in [1] can be extended by replacing $I_{X}$ by $T$ to the following statement: For given LCA groups $\mathbb{G}_{1}$ and $\mathbb{G}_{2}$,

$$
\left\|T\left|\mathcal{F} \mathcal{T}_{p}^{\mathbb{G}_{1}}\|\| I_{\mathbb{C}}\right| \mathcal{F} \mathcal{T}_{p}^{\mathbb{G}_{2}}\right\| \leq\left\|T \mid \mathcal{F} \mathcal{T}_{p}^{\mathbb{G}_{1} \times \mathbb{G}_{2}}\right\|,
$$

where $I_{\mathbb{C}}$ is the identity operator on $\mathbb{C}$. We have $\left\|I_{\mathbb{C}} \mid \mathcal{F} \mathcal{T}_{p}^{\mathbb{G}_{2}}\right\|=1$ when $\mathbb{G}_{2}$ is compact or discrete. Hence if we let $\mathbb{G}_{1}=\mathbb{Z} \times \mathbb{G}$ and $\mathbb{G}_{2}=\mathbb{Z}$ then

$$
\left\|T\left|\mathcal{F} \mathcal{T}_{p}^{\mathbb{Z} \times \mathbb{G}}\|\leq\| T\right| \mathcal{F} \mathcal{T}_{p}^{\mathbb{Z}^{2} \times \mathbb{G}}\right\| .
$$

To show $\left\|T\left|\mathcal{F} \mathcal{T}_{p}^{\mathbb{Z}^{2} \times \mathbb{G}}\|\leq\| T\right| \mathcal{F} \mathcal{T}_{p}^{\mathbb{Z} \times \mathbb{G}}\right\|$ we consider

$$
\mathbf{f}\left(k_{1}, k_{2}, t\right)=\sum_{l_{1}, l_{2}} \mathbf{g}_{l_{1}, l_{2}}(t) \chi_{\left\{l_{1}\right\}}\left(k_{1}\right) \chi_{\left\{l_{2}\right\}}\left(k_{2}\right),
$$

where the summation is on a finite subset of $\mathbb{Z} \times \mathbb{Z}$, and $k_{1}, k_{2} \in \mathbb{Z}, \mathbf{g}_{l_{1}, l_{2}}(t) \in$ $\left[L_{p}(\mathbb{G}), X\right]$. Then

$$
\left\|\mathbf{f} \mid L_{p}\left(\mathbb{Z}^{2} \times \mathbb{G}\right)\right\|=\left(\int_{\mathbb{G} l_{1}, l_{2}}\left\|\mathbf{g}_{l_{1}, l_{2}}(t)\right\|^{p} d t\right)^{1 / p} .
$$

Since

$$
\left[F^{\mathbb{Z}^{2} \times \mathbb{G}}, T\right] \mathbf{f}\left(\widetilde{s}_{1}, \widetilde{s}_{2}, \widetilde{t}\right)=\int_{\mathbb{G}} \sum_{l_{1}, l_{2}} T \mathbf{g}_{l_{1}, l_{2}}(t) \exp \left(i \widetilde{s}_{1} l_{1}\right) \exp \left(i \widetilde{s}_{2} l_{2}\right)(t, \widetilde{t}) d t
$$

we have

$$
\begin{aligned}
& \left\|\left[F^{\mathbb{Z}^{2} \times \mathbb{G}}, T\right] \mathbf{f}\right\|^{p^{\prime}} \\
& =\iint_{\mathbb{G}^{\prime}} \int_{\mathbb{T}}\left\|\int_{\mathbb{G}} \sum_{l_{1}, l_{2}} T \mathbf{g}_{l_{1}, l_{2}}(t) \exp \left(i \widetilde{s}_{1} l_{1}\right) \exp \left(i \widetilde{s}_{2} l_{2}\right)(t, \widetilde{t}) d t\right\|^{p^{\prime}} d \widetilde{s}_{1} d \widetilde{s}_{2} d \widetilde{t} \\
& =\iint_{\mathbb{G}^{\prime}} \int_{\mathbb{T}}\left\|\int_{\mathbb{G}} \sum_{l_{1}, l_{2}} T \mathbf{g}_{l_{1}, l_{2}}(t) \exp \left(i \widetilde{s}_{1} l_{1}\right) \exp \left(i \widetilde{s}_{2} A l_{2}\right)(t, \widetilde{t}) d t\right\|^{p^{\prime}} d \widetilde{s}_{1} d \widetilde{s}_{2} d \widetilde{t}
\end{aligned}
$$

where we have let $A:=2 \max \left\{\left|l_{1}\right|\right\}+1$. Since the Haar measure is translation-invariant we obtain

$$
\begin{aligned}
& \left\|\left[F^{\mathbb{Z}^{2} \times \mathbb{G}}, T\right] \mathbf{f}\right\|^{p^{\prime}} \\
& =\iint_{\mathbb{G}^{\prime}} \int_{\mathbb{T}}\left\|\int_{\mathbb{G}} \sum_{l_{1}, l_{2}} T \mathbf{g}_{l_{1}, l_{2}}(t) \exp \left(i \widetilde{s}_{1} l_{1}\right) \exp \left(i\left(\widetilde{s}_{1}+\widetilde{s}_{2}\right) A l_{2}\right)(t, \widetilde{t}) d t\right\|^{p^{\prime}} d \widetilde{s}_{1} d \widetilde{s}_{2} d \widetilde{t} \\
& =\iint_{\mathbb{G}^{\prime}} \int_{\mathbb{T}}\left\|\int_{\mathbb{G}} \sum_{l_{1}, l_{2}} T \mathbf{g}_{l_{1}, l_{2}}(t) \exp \left(i \widetilde{s}_{1}\left(l_{1}+A l_{2}\right)\right) \exp \left(i \widetilde{s}_{2} A l_{2}\right)(t, \widetilde{t}) d t\right\|^{p^{\prime}} d \widetilde{s}_{1} d \widetilde{s}_{2} d \widetilde{t} \\
& =\iint_{\mathbb{T}} \int_{\mathbb{G}^{\prime} \mathbb{T}}\left\|\int_{\mathbb{G}} \sum_{l_{1}, l_{2}} T\left(\mathbf{g}_{l_{1}, l_{2}}(t) \exp \left(i \widetilde{s}_{2} A l_{2}\right)\right) \exp \left(i \widetilde{s}_{1}\left(l_{1}+A l_{2}\right)\right)(t, \widetilde{t}) d t\right\|^{p^{\prime}} d \widetilde{s}_{1} d \widetilde{t} d \widetilde{s}_{2} .
\end{aligned}
$$


Since $\left(l_{1}, l_{2}\right) \mapsto l_{1}+A l_{2}$ is a one-to-one correspondence, the above equals

$$
\begin{aligned}
\int_{\mathbb{T}} \|\left[F^{\mathbb{Z} \times \mathbb{G}}, T\right]\left(\sum_{l_{1}+A l_{2}}\left(\mathbf{g}_{l_{1}, l_{2}}(t) \exp \left(i \widetilde{s}_{2} A l_{2}\right)\right) \chi_{\left\{l_{1}+A l_{2}\right\}} \|^{p^{\prime}} d \widetilde{s}_{2}\right. \\
\quad \leq \int_{\mathbb{T}}\left[\left\|T \mid \mathcal{F} \mathcal{T}_{p}^{\mathbb{Z} \times \mathbb{G}}\right\|^{p^{\prime}}\left(\int_{\mathbb{G} l_{1}+A l_{2}}\left\|\mathbf{g}_{l_{1}, l_{2}}(t) \exp \left(i \widetilde{s}_{2} A l_{2}\right)\right\|^{p} d t\right)^{p^{\prime} / p}\right] d \widetilde{s}_{2} \\
\quad=\left\|T \mid \mathcal{F} \mathcal{T}_{p}^{\mathbb{Z} \times \mathbb{G}}\right\|^{p^{\prime}}\left(\int_{\mathbb{G}} \sum_{l_{1}+A l_{2}}\left\|\mathbf{g}_{l_{1}, l_{2}}(t)\right\|^{p} d t\right)^{p^{\prime} / p} \\
=\left\|T \mid \mathcal{F} \mathcal{T}_{p}^{\mathbb{Z} \times \mathbb{G}}\right\|^{p^{\prime}}\left(\int_{\mathbb{G} l_{1}, l_{2}}\left\|\mathbf{g}_{l_{1}, l_{2}}(t)\right\|^{p} d t\right)^{p^{\prime} / p} \\
\quad=\left\|T\left|\mathcal{F} \mathcal{T}_{p}^{\mathbb{Z} \times \mathbb{G}}\left\|^{p^{\prime}}\right\| \mathbf{f}\right| L_{p}\left(\mathbb{Z}^{2} \times \mathbb{G}, X\right)\right\|^{p^{\prime}} \quad \text { by }(3),
\end{aligned}
$$

so it follows that $\left\|T\left|\mathcal{F} \mathcal{T}_{p}^{\mathbb{Z}^{2} \times \mathbb{G}}\|\leq\| T\right| \mathcal{F} \mathcal{T}_{p}^{\mathbb{Z} \times \mathbb{G}}\right\|$.

REMARK 1. In fact, every dissipative group $\mathbb{A}$ has the property that $\left\|T\left|\mathcal{F} \mathcal{T}_{p}^{\mathbb{A}^{n} \times \mathbb{G}}\|=\| T\right| \mathcal{F} \mathcal{T}_{p}^{\mathbb{A} \times \mathbb{G}}\right\|$ for any positive integer $n$ and for any LCA group $\mathbb{G}$. The definition and properties of dissipative groups are found in [4].

Corollary 6. $\left\|T\left|\mathcal{F} \mathcal{T}_{p}^{\mathbb{T}^{n} \times \mathbb{G}}\|=\| T\right| \mathcal{F} \mathcal{T}_{p}^{\mathbb{T} \times \mathbb{G}}\right\|$ and so $\mathcal{F T}_{p}^{\mathbb{T}^{n} \times \mathbb{G}}=\mathcal{F T}_{p}^{\mathbb{T} \times \mathbb{G}}$.

Proof. This follows from Lemma 5 and a duality argument.

Proposition 7. Let $\mathbb{H}$ be $\mathbb{R}, \mathbb{Z}$ or $\mathbb{T}$, and $\mathbb{G}$ be an LCA group. Then $\mathcal{F} \mathcal{T}_{p}^{\mathbb{H}^{n} \times \mathbb{G}}=\mathcal{F} \mathcal{T}_{p}^{\mathbb{Z} \times \mathbb{G}}$ for any integer $n \geq 1$. In particular, $\mathcal{F} \mathcal{T}_{p}^{\mathbb{H}^{n}}=\mathcal{F} \mathcal{T}_{p}^{\mathbb{Z}}$.

Proof. It follows from Corollary 4 and Lemma 5 that $\mathcal{F} \mathcal{T}_{p}^{\mathbb{H}^{n} \times \mathbb{G}}=\mathcal{F} \mathcal{T}_{p}^{\mathbb{Z} \times \mathbb{G}}$. And if $\mathbb{G}$ is the trivial group then we have $\mathcal{F} \mathcal{T}_{p}^{\mathbb{H}^{n}}=\mathcal{F} \mathcal{T}_{p}^{\mathbb{Z}}$.

Notation: From now on, when $\mathbb{H}$ is $\mathbb{R}, \mathbb{Z}$ or $\mathbb{T}$ we denote $\mathcal{F} \mathcal{T}_{p}^{\mathbb{H}}$ by $\mathcal{F} \mathcal{T}_{p}$.

COROLlARY 8. $\mathcal{F} \mathcal{T}_{p}^{\mathbb{R}^{a} \times \mathbb{Z}^{b} \times \mathbb{T}^{c}}=\mathcal{F} \mathcal{T}_{p}$ for any nonnegative integers $a, b, c$ with $a+b+c \geq 1$.

Proof. Without loss of generality we assume $a, b, c>0$. By applying Proposition 7, we have

$$
\mathcal{F} \mathcal{T}_{p}^{\mathbb{R}^{a} \times \mathbb{Z}^{b} \times \mathbb{T}^{c}}=\mathcal{F} \mathcal{T}_{p}^{\mathbb{Z}^{b+1} \times \mathbb{T}^{c}}=\mathcal{F} \mathcal{T}_{p}^{\mathbb{Z} \times \mathbb{T}^{c}}=\mathcal{F} \mathcal{T}_{p}^{\mathbb{Z}^{2}}=\mathcal{F} \mathcal{T}_{p}^{\mathbb{Z}}
$$

TheOREM 9. Let $\mathbb{G}$ be an LCA group. Then $\mathcal{F} \mathcal{T}_{p}^{\mathbb{R}^{a} \times \mathbb{Z}^{b} \times \mathbb{T}^{c} \times \mathbb{G}}=\mathcal{F} \mathcal{T}_{p}^{\mathbb{Z} \times \mathbb{G}}$ for any nonnegative integers $a, b, c$ with $a+b+c \geq 1$.

Proof. By Proposition 7 we have

$$
\mathcal{F} \mathcal{T}_{p}^{\mathbb{R}^{a} \times \mathbb{Z}^{b} \times \mathbb{T}^{c} \times \mathbb{G}}=\mathcal{F} \mathcal{T}_{p}^{\mathbb{Z}^{1+b} \times \mathbb{T}^{c} \times \mathbb{G}}=\mathcal{F} \mathcal{T}_{p}^{\mathbb{Z}^{2+b} \times \mathbb{G}}=\mathcal{F} \mathcal{T}_{p}^{\mathbb{Z} \times \mathbb{G}}
$$




\section{Search for $\mathbb{G}$ satisfying $\mathcal{F} \mathcal{T}_{p}^{\mathbb{G}}=\mathcal{F} \mathcal{T}_{p}^{\mathbb{Z}}$ and further classification.}

We notice that to solve the problem of deciding whether $\mathcal{F} \mathcal{T}_{p}^{\mathbb{G}}$ depends on $\mathbb{G}$, we should solve that problem for the compact abelian groups since any compactly generated LCA group is a product of a finite number of $\mathbb{R}$ 's and $\mathbb{Z}$ 's and a compact group [5]. The dual group of a compact group has the discrete topology [13]. Moreover if $\mathbb{G}$ is a compact abelian group then $\mathbb{G}$ is connected iff $\mathbb{G}^{\prime}$ is torsion-free [5]. This fact gives a clue to the results in this section.

First, we introduce Weil's formula which factorizes integration on $\mathbb{G}$ into double integration on a closed subgroup and its factor group.

TheOREM 10 ([12]). Let $\mathbb{G}$ be an LCA group and $\mathbb{H}$ a closed subgroup. Then there are Haar measures $\mu_{\mathbb{G}}, \mu_{\mathbb{H}}$ and $\mu_{\mathbb{G} / \mathbb{H}}$ such that

$$
\int_{\mathbb{G}} \mathbf{f}(s) d_{\mu_{\mathbb{G}}}(s)=\int_{\mathbb{G} / \mathbb{H}}\left(\int_{\mathbb{H}} \mathbf{f}(s+h) d_{\mu_{\mathbb{H}}}(h)\right) d_{\mu_{\mathbb{G}} / \mathbb{H}}(s+\mathbb{H})
$$

whenever $\mathbf{f}$ is a compactly supported continuous Banach space-valued function or a nonnegative lower semicontinuous function on $\mathbb{G}$.

In Theorem 10 if any two of $\mu_{\mathbb{G}}, \mu_{\mathbb{H}}$ and $\mu_{\mathbb{G} / \mathbb{H}}$ are given then the third can be determined so that the statement holds.

Andersson [1] obtained the inequality $\left\|I_{X}\left|\mathcal{F} \mathcal{T}_{p}^{\mathbb{H}}\|\leq\| I_{X}\right| \mathcal{F} \mathcal{T}_{p}^{\mathbb{G}}\right\|$, where $\mathbb{H}$ is an open subgroup of an LCA group $\mathbb{G}$ and $I_{X}$ is the identity operator on a Banach space $X$. By replacing $I_{X}$ with a bounded linear operator $T: X \rightarrow Y$ in the proof of [1], we have the following:

Proposition 11. Let $\mathbb{H}$ be an open subgroup of an LCA group $\mathbb{G}$. Then

$$
\left\|T\left|\mathcal{F} \mathcal{T}_{p}^{\mathbb{H}}\|\leq\| T\right| \mathcal{F} \mathcal{T}_{p}^{\mathbb{G}}\right\|
$$

Now we consider torsion-free LCA groups with the discrete topology.

COROLlary 12. For any nontrivial torsion-free LCA group $\mathbb{E}$ with the discrete topology,

$$
\left\|T\left|\mathcal{F} \mathcal{T}_{p}^{\mathbb{E}}\|=\| T\right| \mathcal{F} \mathcal{T}_{p}^{\mathbb{Z}}\right\|
$$

Proof. The proof is similar to that of $\mathrm{FT}_{p}^{\mathbb{E}}=\mathrm{FT}_{p}^{\mathbb{Z}}$ in [1]: Since $\mathbb{Z}$ is isomorphic to an open subgroup of $\mathbb{E}$, by applying (5) we have $\left\|T \mid \mathcal{F} \mathcal{T}_{p}^{\mathbb{Z}}\right\| \leq$ $\left\|T \mid \mathcal{F} \mathcal{T}_{p}^{\mathbb{E}}\right\|$. On the other hand, for any $X$-valued simple function $\mathbf{f}$ defined on $\mathbb{E}$ which has finite $L_{p}$-norm, $\mathbf{f}$ is nonzero only on a subset of an open subgroup which is isomorphic to $\mathbb{Z}^{k}$ for some positive integer $k$. Therefore we have

$$
\frac{\left\|\left[F^{\mathbb{E}}, T\right] \mathbf{f}\right\|_{p^{\prime}}}{\left\|\mathbf{f} \mid L_{p}(\mathbb{E})\right\|}=\frac{\left\|\left[F^{\mathbb{Z}^{k}}, T\right] \mathbf{f}\right\|_{p^{\prime}}}{\left\|\mathbf{f} \mid L_{p}\left(\mathbb{Z}^{k}\right)\right\|} \leq\left\|T \mid \mathcal{F} \mathcal{T}_{p}^{\mathbb{Z}^{k}}\right\|
$$


and hence

$$
\left\|T\left|\mathcal{F} \mathcal{T}_{p}^{\mathbb{E}}\left\|\leq \sup _{k}\right\| T\right| \mathcal{F} \mathcal{T}_{p}^{\mathbb{Z}^{k}}\right\|=\left\|T \mid \mathcal{F} \mathcal{T}_{p}^{\mathbb{Z}}\right\|
$$

Thus equality (6) follows.

Lemma 13. Let $\mathbb{H}$ be a closed subgroup of an LCA group $\mathbb{G}$ such that $\mathbb{G} / \mathbb{H}$ is finite. If $n$ is the cardinality of $\mathbb{G} / \mathbb{H}$ then

$$
\left\|T\left|\mathcal{F} \mathcal{T}_{p}^{\mathbb{H}}\|\leq\| T\right| \mathcal{F} \mathcal{T}_{p}^{\mathbb{G}}\right\| \leq n^{1 / p^{\prime}}\left\|T \mid \mathcal{F} \mathcal{T}_{p}^{\mathbb{H}}\right\|,
$$

and hence $\mathcal{F} \mathcal{T}_{p}^{\mathbb{G}}=\mathcal{F} \mathcal{T}_{p}^{\mathbb{H}}$.

Proof. Since $\mathbb{G} / \mathbb{H}$ is finite, $\mathbb{H}$ is open and by (5) we have the left inequality. The proof of the right one is as follows. Choose $s_{1}, \ldots, s_{n}$ in $\mathbb{G}$ such that $\mathbb{G} / \mathbb{H}=\left\{s_{1}+\mathbb{H}, \ldots, s_{n}+\mathbb{H}\right\}$. The measure of $\mathbb{G} / \mathbb{H}$ is the Haar measure of unit mass. By applying Weil's formula, for any compactly supported continuous $X$-valued function $\mathbf{f}$ on $\mathbb{G}$ we have

$$
\begin{aligned}
\int_{\mathbb{G}}\|\mathbf{f}(s)\|^{p} d_{\mu_{\mathbb{G}}}(s) & =\int_{\mathbb{G} / \mathbb{H}}\left(\int_{\mathbb{H}}\|\mathbf{f}(s+h)\|^{p} d_{\mu_{\mathbb{H}}}(h)\right) d_{\mu_{\mathbb{G} / \mathbb{H}}}(s+\mathbb{H}) \\
& =\sum_{i=1}^{n} \frac{1}{n} \int_{\mathbb{H}}\left\|\mathbf{f}\left(s_{i}+h\right)\right\|^{p} d_{\mu_{\mathbb{H}}}(h)
\end{aligned}
$$

and

$$
\begin{aligned}
{\left[F^{\mathbb{G}}, T\right] \mathbf{f}(\sigma) } & =\sum_{i=1}^{n} \frac{1}{n}\left(\int_{\mathbb{H}} T \mathbf{f}\left(s_{i}+h\right) \sigma\left(s_{i}+h\right) d_{\mu_{\mathbb{H}}}(h)\right) \\
& =\sum_{i=1}^{n} \frac{\sigma\left(s_{i}\right)}{n}\left(\int_{\mathbb{H}} T \mathbf{f}\left(s_{i}+h\right) \sigma(h) d_{\mu_{\mathbb{H}}}(h)\right)
\end{aligned}
$$

for $\sigma \in \mathbb{G}^{\prime}$. The dual group of $\mathbb{G} / \mathbb{H}$ is isomorphic to the closed subgroup $\mathbb{H}^{\perp}=\left\{\chi \in \mathbb{G}^{\prime} \mid \chi(h)=1\right.$ for all $\left.h \in \mathbb{H}\right\}$, and $\mathbb{G}^{\prime} / \mathbb{H}^{\perp}$ is isomorphic to $\mathbb{H}^{\prime}$. Here the cardinality of $\mathbb{H}^{\perp}$ is $n$, the measure of $\mathbb{H}^{\perp}$ is the counting measure, and we write $\mathbb{H}^{\perp}$ as $\left\{\widetilde{\eta}_{1}, \ldots, \widetilde{\eta}_{n}\right\}$. Now $\left[F^{\mathbb{G}}, T\right](\mathbf{f})$ belongs to $\left[C_{0}\left(\mathbb{G}^{\prime}\right), Y\right]$, so the $Y$-norm of $\left[F^{\mathbb{G}}, T\right](\mathbf{f})$ is continuous and by Weil's formula we have

$$
\begin{aligned}
& \left\|\left[F^{\mathbb{G}}, T\right] \mathbf{f}\right\| \\
& =\left(\int_{\mathbb{H}^{\prime}} \sum_{j=1}^{n}\left\|\frac{1}{n} \sum_{i=1}^{n} \sigma\left(s_{i}\right) \eta_{j}\left(s_{i}\right) \int_{\mathbb{H}} T \mathbf{f}\left(s_{i}+h\right) \sigma(h) d_{\mu_{\mathbb{H}}}(h)\right\|^{p^{\prime}} d_{\mu_{\mathbb{H}^{\prime}}}\left(\sigma+\mathbb{H}^{\perp}\right)\right)^{1 / p^{\prime}} \\
& \leq\left[\left(\int_{\mathbb{H}^{\prime}} \sum_{j=1}^{n}\left(\sum_{i=1}^{n} \frac{1}{n}\left\|\int_{\mathbb{H}} T \mathbf{f}\left(s_{i}+h\right) \sigma(h) d_{\mu_{\mathbb{H}}}(h)\right\|^{p}\right)^{p^{\prime} / p} d_{\mu_{\mathbb{H}^{\prime}}}\left(\sigma+\mathbb{H}^{\perp}\right)\right)^{p / p^{\prime}}\right]^{1 / p}
\end{aligned}
$$




$$
\begin{aligned}
& \leq\left[\sum_{i=1}^{n} \frac{1}{n}\left(\int_{\mathbb{H} \mathbb{H}^{\prime}} \sum_{j=1}^{n}\left\|\int_{\mathbb{H}} T \mathbf{f}\left(s_{i}+h\right) \sigma(h) d_{\mu_{\mathbb{H}}}(h)\right\|^{p^{\prime}} d_{\mu_{\mathbb{H}^{\prime}}}\left(\sigma+\mathbb{H}^{\perp}\right)\right)^{p / p^{\prime}}\right]^{1 / p} \\
& =\left[\sum_{i=1}^{n} \frac{1}{n} n^{p / p^{\prime}}\left(\int_{\mathbb{H}^{\prime}}\left\|\int_{\mathbb{H}} T \mathbf{f}\left(s_{i}+h\right) \sigma(h) d_{\mu_{\mathbb{H}}}(h)\right\|^{p^{\prime}} d_{\mu_{\mathbb{H}^{\prime}}}\left(\sigma+\mathbb{H}^{\perp}\right)\right)^{p / p^{\prime}}\right]^{1 / p} \\
& \leq\left[\sum_{i=1}^{n} \frac{1}{n} n^{p / p^{\prime}}\left\|T \mid \mathcal{F} \mathcal{T}_{p}^{\mathbb{H}}\right\|^{p}\left(\int_{\mathbb{H}}\left\|\mathbf{f}\left(s_{i}+h\right)\right\|^{p} d_{\mu_{\mathbb{H}}}(h)\right)\right]^{1 / p} \\
& =n^{1 / p^{\prime}}\left\|T\left|\mathcal{F} \mathcal{T}_{p}^{\mathbb{H}}\|\| \mathbf{f}\right| L_{p}(\mathbb{G})\right\| .
\end{aligned}
$$

We have used Minkowski's inequality in the third inequality above.

Proposition 14. If $\mathbb{F}$ is an infinite, compact and connected LCA group then for any nonnegative integers $a, b$, there exist $c(a, b), C(a, b)>0$ such that

$$
c(a, b)\left\|T\left|\mathcal{F} \mathcal{T}_{p}^{\mathbb{T}}\|\leq\| T\right| \mathcal{F} \mathcal{T}_{p}^{\mathbb{R}^{a} \times \mathbb{Z}^{b} \times \mathbb{F}}\right\| \leq C(a, b)\left\|T \mid \mathcal{F} \mathcal{T}_{p}^{\mathbb{T}}\right\|
$$

Therefore $\mathcal{F} \mathcal{T}_{p}^{\mathbb{R}^{a} \times \mathbb{Z}^{b} \times \mathbb{F}}=\mathcal{F} \mathcal{T}_{p}$.

Proof. If $a=b=0$ then by applying (6) we have $\left\|T^{\prime}\left|\mathcal{F} \mathcal{T}_{p}^{\mathbb{F}^{\prime}}\|=\| T^{\prime}\right| \mathcal{F} \mathcal{T}_{p}^{\mathbb{Z}}\right\|$, where $\mathbb{F}^{\prime}$ is the dual group of $\mathbb{F}$ and hence a nontrivial torsion-free group with the discrete topology. It follows that

$$
\left\|T\left|\mathcal{F} \mathcal{T}_{p}^{\mathbb{F}}\|=\| T^{\prime}\right| \mathcal{F} \mathcal{T}_{p}^{\mathbb{Z}}\right\|=\left\|T \mid \mathcal{F} \mathcal{T}_{p}^{\mathbb{T}}\right\| \quad \text { and } \quad \mathcal{F} \mathcal{T}_{p}^{\mathbb{F}}=\mathcal{F} \mathcal{T}_{p}
$$

If $a+b \geq 1$ then by applying Theorem 9 and Corollary 4 , we have

$$
\mathcal{F} \mathcal{T}_{p}^{\mathbb{R}^{a} \times \mathbb{Z}^{b} \times \mathbb{F}}=\mathcal{F} \mathcal{T}_{p}^{\mathbb{T} \times \mathbb{F}}
$$

and in fact

$$
c(a, b)\left\|T\left|\mathcal{F} \mathcal{T}_{p}^{\mathbb{T} \times \mathbb{F}}\|\leq\| T\right| \mathcal{F} \mathcal{T}_{p}^{\mathbb{R}^{a} \times \mathbb{Z}^{b} \times \mathbb{F}}\right\| \leq C(a, b)\left\|T \mid \mathcal{F} \mathcal{T}_{p}^{\mathbb{T} \times \mathbb{F}}\right\|
$$

for some positive reals $c(a, b), C(a, b)$. Here $c(a, b)=(2 / \pi)^{a b}, C(a, b)=1$. Note that $\mathbb{T} \times \mathbb{F}$ is connected and compact. Thus we again have

$$
\left\|T\left|\mathcal{F} \mathcal{T}_{p}^{\mathbb{T} \times \mathbb{F}}\|=\| T\right| \mathcal{F} \mathcal{T}_{p}^{\mathbb{T}}\right\| \quad \text { by }(10)
$$

If $\mathbb{F}$ has only finitely many components and $\mathbb{F}_{0}$ is the component (maximal connected set) of the identity element (briefly, the identity component), then $\mathbb{F} / \mathbb{F}_{0}$ is finite and we have the following result.

THEOREM 15. Let $\mathbb{F}$ be an infinite compact LCA group with $n$ components. Then for any nonnegative integers $a, b$, there exist $c(a, b), C(a, b)>0$ such that

$$
c(a, b)\left\|T\left|\mathcal{F} \mathcal{T}_{p}^{\mathbb{T}}\|\leq\| T\right| \mathcal{F} \mathcal{T}_{p}^{\mathbb{R}^{a} \times \mathbb{Z}^{b} \times \mathbb{F}}\right\| \leq n^{1 / p^{\prime}} C(a, b)\left\|T \mid \mathcal{F} \mathcal{T}_{p}^{\mathbb{T}}\right\|,
$$

and therefore $\mathcal{F} \mathcal{T}_{p}^{\mathbb{R}^{a} \times \mathbb{Z}^{b} \times \mathbb{F}}=\mathcal{F} \mathcal{T}_{p}$. 
Proof. Let $\mathbb{F}_{0}$ be the identity component of $\mathbb{F}$. Then the factor group $\left(\mathbb{R}^{a} \times \mathbb{Z}^{b} \times \mathbb{F}\right) /\left(\mathbb{R}^{a} \times \mathbb{Z}^{b} \times \mathbb{F}_{0}\right)$ is a finite group with $n$ elements. Thus by Lemma 13 ,

$$
\left\|T\left|\mathcal{F} \mathcal{T}_{p}^{\mathbb{R}^{a} \times \mathbb{Z}^{b} \times \mathbb{F}_{0}}\|\leq\| T\right| \mathcal{F} \mathcal{T}_{p}^{\mathbb{R}^{a} \times \mathbb{Z}^{b} \times \mathbb{F}_{0}}\right\| \leq n^{1 / p^{\prime}}\left\|T \mid \mathcal{F} \mathcal{T}_{p}^{\mathbb{R}^{a} \times \mathbb{Z}^{b} \times \mathbb{F}}\right\|
$$

and

$$
\mathcal{F} \mathcal{T}_{p}^{\mathbb{R}^{a} \times \mathbb{Z}^{b} \times \mathbb{F}}=\mathcal{F} \mathcal{T}_{p}^{\mathbb{R}^{a} \times \mathbb{Z}^{b} \times \mathbb{F}_{0}} .
$$

Then the statement follows from Proposition 14.

This theorem cannot say anything about the case when $\mathbb{F}$ has infinitely many components, because of the factor $n^{1 / p^{\prime}}$.

Theorem 15 can be extended beyond the scope of compactly generated LCA groups.

THEOREM 16. Let $\mathbb{E}$ be a nontrivial torsion-free group with the discrete topology and $\mathbb{F}$ an infinite, compact and connected LCA group. Then for any LCA group $\mathbb{G}$,

$$
\left\|T\left|\mathcal{F} \mathcal{T}_{p}^{\mathbb{E} \times \mathbb{G}}\|=\| T\right| \mathcal{F} \mathcal{T}_{p}^{\mathbb{Z} \times \mathbb{G}}\right\|, \quad\left\|T\left|\mathcal{F} \mathcal{T}_{p}^{\mathbb{F} \times \mathbb{G}}\|=\| T\right| \mathcal{F} \mathcal{T}_{p}^{\mathbb{T} \times \mathbb{G}}\right\| .
$$

If $\widetilde{\mathbb{F}}$ is a compact $L C A$ group with $n$ components then for any nonnegative integer $k$ there exist $c(k), C(k)$ such that

$$
c(k)\left\|T\left|\mathcal{F} \mathcal{T}_{p}^{\mathbb{T} \times \mathbb{G}}\|\leq\| T\right| \mathcal{F} \mathcal{T}_{p}^{\mathbb{R}^{k} \times \mathbb{E}^{l} \times \widetilde{\mathbb{F}}^{m} \times \mathbb{G}}\right\| \leq n^{m / p^{\prime}} C(k)\left\|T \mid \mathcal{F} \mathcal{T}_{p}^{\mathbb{T} \times \mathbb{G}}\right\|,
$$

and hence

$$
\mathcal{F} \mathcal{T}_{p}^{\mathbb{R}^{k} \times \mathbb{E}^{l} \times \widetilde{\mathbb{F}}^{m} \times \mathbb{G}}=\mathcal{F} \mathcal{T}_{p}^{\mathbb{T} \times \mathbb{G}},
$$

where $l, m=0$ or 1 and $k+l+m \geq 1$. In particular if $\mathbb{G}$ is the trivial group then

$$
\mathcal{F} \mathcal{T}_{p}^{\mathbb{R}^{k} \times \mathbb{E}^{l} \times \widetilde{\mathbb{F}}^{m}}=\mathcal{F} \mathcal{T}_{p}
$$

Proof. First, $\mathbb{Z}$ is isomorphic to an open subgroup of $\mathbb{E}$, and so $\mathbb{Z} \times \mathbb{G}$ is an open subgroup of $\mathbb{E} \times \mathbb{G}$. By applying (5) we have $\left\|T \mid \mathcal{F} \mathcal{T}_{p}^{\mathbb{Z} \times \mathbb{G}}\right\| \leq$ $\left\|T \mid \mathcal{F} \mathcal{T}_{p}^{\mathbb{E} \times \mathbb{G}}\right\|$. Conversely, for any simple $X$-valued function $\mathbf{f}$ which is defined on $\mathbb{E} \times \mathbb{G}$ and has finite $L_{p}$-norm, the support of $\mathbf{f}$ is a subset of $\mathbb{Z}^{a} \times \mathbb{G}$ for some positive integer $a$. Therefore Lemma 5 yields $\left\|T \mid \mathcal{F} \mathcal{T}_{p}^{\mathbb{E} \times \mathbb{G}}\right\| \leq$ $\sup _{a}\left\|T\left|\mathcal{F} \mathcal{T}_{p}^{\mathbb{Z}^{a} \times \mathbb{G}}\|=\| T\right| \mathcal{F} \mathcal{T}_{p}^{\mathbb{Z} \times \mathbb{G}}\right\|$.

Second, from the duality argument it follows that

$$
\left\|T\left|\mathcal{F} \mathcal{T}_{p}^{\mathbb{F} \times \mathbb{G}}\|=\| T^{\prime}\right| \mathcal{F} \mathcal{T}_{p}^{\mathbb{F}^{\prime} \times \mathbb{G}^{\prime}}\right\|=\left\|T^{\prime}\left|\mathcal{F} \mathcal{T}_{p}^{\mathbb{Z} \times \mathbb{G}^{\prime}}\|=\| T\right| \mathcal{F} \mathcal{T}_{p}^{\mathbb{T} \times \mathbb{G}}\right\| .
$$

The rest follows by applying the above two results, Propositions 1, 2 and Lemma 13.

REMARK 2. If $\mathbb{G}$ is an LCA group and $\mathbb{G}_{0}$ is the identity component of $\mathbb{G}$ then $\mathbb{G}_{0}$ is a closed normal subgroup of $\mathbb{G}$ and the factor group $\mathbb{G} / \mathbb{G}_{0}$ is totally disconnected and Hausdorff. From $(24.45)$ of [5], if $\mathbb{G}_{0}$ is open then 
$\mathbb{G}$ is isomorphic to $\mathbb{G}_{0} \times\left(\mathbb{G} / \mathbb{G}_{0}\right)$. In particular $\mathbb{G}_{0}$ is open when $\mathbb{G}$ is locally connected. Hence to answer the question, denoted by $(\mathrm{P})$, whether $\left\|T \mid \mathcal{F} \mathcal{T}_{p}^{\mathbb{G}}\right\|$ is equivalent to $\left\|T \mid \mathcal{F} \mathcal{T}_{p}^{\mathbb{T}}\right\|$ or not, it is useful to find $\mathbb{G}_{0}$ and $\mathbb{G} / \mathbb{G}_{0}$. Since $\mathbb{G}_{0}$ is isomorphic to $\mathbb{R}^{n} \times \mathbb{K}$, where $n$ is a nonnegative integer and $\mathbb{K}$ is a compact connected group (see Theorem 9.14 of [5]), the answer to (P) is affirmative when $\mathbb{G} / \mathbb{G}_{0}$ is good.

TheOREm 17. Let $\mathbb{G}$ be an LCA group with $n$ components. Then there are positive real numbers $c$ and $C$ such that

$$
c\left\|T\left|\mathcal{F} \mathcal{T}_{p}^{\mathbb{T}}\|\leq\| T\right| \mathcal{F} \mathcal{T}_{p}^{\mathbb{G}}\right\| \leq n^{1 / p^{\prime}} C\left\|T \mid \mathcal{F} \mathcal{T}_{p}^{\mathbb{T}}\right\|,
$$

and hence $\mathcal{F} \mathcal{T}_{p}^{\mathbb{G}}=\mathcal{F} \mathcal{T}_{p}$.

Proof. Let $\mathbb{G}_{0}$ be the identity component of $\mathbb{G}$. Then $\mathbb{G} / \mathbb{G}_{0}$ is a finite LCA group. Hence by Lemma 13 we have $\left\|T\left|\mathcal{F} \mathcal{T}_{p}^{\mathbb{G}_{0}}\|\leq\| T\right| \mathcal{F} \mathcal{T}_{p}^{\mathbb{G}}\right\| \leq$ $n^{1 / p^{\prime}}\left\|T \mid \mathcal{F} \mathcal{T}_{p}^{\mathbb{G}_{0}}\right\|$. Moreover, $\mathbb{G}_{0}$ is isomorphic to $\mathbb{R}^{k} \times \mathbb{K}$, where $k$ is a nonnegative integer and $\mathbb{K}$ is a compact connected group. Now, $\mathbb{K}$ is trivial or infinite. If $\mathbb{K}$ is trivial then from Proposition 2 it follows that

$$
c\left\|T\left|\mathcal{F} \mathcal{T}_{p}^{\mathbb{T}^{k}}\|\leq\| T\right| \mathcal{F} \mathcal{T}_{p}^{\mathbb{G}_{0}}\right\| \leq C\left\|T \mid \mathcal{F} \mathcal{T}_{p}^{\mathbb{T}^{k}}\right\|
$$

for some $c, C>0$. Moreover, by (10), $\left\|T\left|\mathcal{F} \mathcal{T}_{p}^{\mathbb{T}^{k}}\|=\| T\right| \mathcal{F} \mathcal{T}_{p}^{\mathbb{T}}\right\|$. Thus we have

$$
c\left\|T\left|\mathcal{F} \mathcal{T}_{p}^{\mathbb{T}}\|\leq\| T\right| \mathcal{F} \mathcal{T}_{p}^{\mathbb{G}_{0}}\right\| \leq C\left\|T \mid \mathcal{F} \mathcal{T}_{p}^{\mathbb{T}}\right\| .
$$

If $\mathbb{K}$ is infinite, we also have (12) by Proposition 14 . Therefore the inequality (11) follows, and $\mathcal{F} \mathcal{T}_{p}^{\mathbb{G}}=\mathcal{F} \mathcal{T}_{p}$.

THEOREM 18. If $\mathbb{G}_{0}$ is the identity component of an LCA group $\mathbb{G}$ and $\mathbb{G} / \mathbb{G}_{0}$ is the direct sum of a nontrivial discrete torsion-free group and a finite group with cardinality $n$, then $\left\|T\left|\mathcal{F} \mathcal{T}_{p}^{\mathbb{G}_{0}}\|\leq\| T\right| \mathcal{F} \mathcal{T}_{p}^{\mathbb{G}}\right\| \leq n^{1 / p^{\prime}}\left\|T \mid \mathcal{F} \mathcal{T}_{p}^{\mathbb{G}_{0} \times \mathbb{Z}}\right\|$ and $\mathcal{F} \mathcal{T}_{p}^{\mathbb{G}}=\mathcal{F} \mathcal{T}_{p}$.

Proof. $\mathbb{G}$ is isomorphic to $\mathbb{G}_{0} \times \mathbb{G} / \mathbb{G}_{0}$ because $\mathbb{G}_{0}$ is open. The direct sum of a nontrivial discrete torsion-free group and a finite group is isomorphic to the direct product of the two. We can apply Proposition 11, Lemma 13 and Theorem 16 to obtain the assertion.

As for the class of Banach spaces which have Fourier type $p$ for an infinite LCA group, we have the following:

COROLlary 19. (i) Under the assumptions of Theorem 16,

$$
\mathrm{FT}_{p}^{\mathbb{R}^{k} \times \mathbb{E}^{l} \times \widetilde{\mathbb{F}}^{m}}=\mathrm{FT}_{p}
$$

(ii) Under the assumptions of Theorem 18,

$$
\mathrm{FT}_{p}^{\mathbb{G}}=\mathrm{FT}_{p}
$$


Hinrichs and Lee in [6], by combining the results of Propositions 1, 2 and Lemma 5 of this paper with the structure of LCA groups, prove that $\mathcal{F} \mathcal{T}_{p}^{\mathbb{G}}=\mathcal{F} \mathcal{T}_{p}^{\mathbb{G}^{\prime}}$ for any LCA group $\mathbb{G}$ and $1<p \leq 2$. They derive the general statement:

Let $\mathbb{G}_{1}, \mathbb{G}_{2}$ and $\mathbb{H}$ be LCA groups and $1<p \leq 2$. If there exists a constant $c$ such that $\left\|T\left|\mathcal{F} \mathcal{T}_{p}^{\mathbb{G} 1}\|\leq c\| T\right| \mathcal{F} \mathcal{T}_{p}^{\mathbb{G}_{2}}\right\|$ for all operators $T \in \mathcal{F} \mathcal{T}_{p}^{\mathbb{G}_{2}}$ then also $\left\|T\left|\mathcal{F} \mathcal{T}_{p}^{\mathbb{G}_{1} \times \mathbb{H}}\|\leq c\| T\right| \mathcal{F} \mathcal{T}_{p}^{\mathbb{G}_{2} \times \mathbb{H}}\right\|$ for all operators $T \in \mathcal{F} \mathcal{T}_{p}^{\mathbb{G}_{2} \times \mathbb{H}}$.

This statement, combined with the inequalities

$$
\left\|T\left|\mathcal{F} \mathcal{T}_{p}^{\mathbb{R}}\|\leq\| T\right| \mathcal{F} \mathcal{T}_{p}^{\mathbb{F}}\right\|=\left\|T\left|\mathcal{F} \mathcal{T}_{p}^{\mathbb{Z}}\left\|\leq \frac{\pi}{2}\right\| T\right| \mathcal{F} \mathcal{T}_{p}^{\mathbb{R}}\right\|,
$$

where $\mathbb{F}$ is a torsion-free group, implies Propositions 1, 2 and Lemma 5.

Acknowledgements. The author would like to thank Professor Chansun Choi and Hun Hee Lee for their kind advice and comments. The author would also like to thank the anonymous referee for the helpful comments.

\section{References}

[1] M. E. Andersson, On the vector valued Hausdorff-Young inequality, Ark. Mat. 36 (1998), 1-30.

[2] J. Bourgain, Vector-valued Hausdorff-Young inequalities and applications, in: Geometric Aspects of Functional Analysis, Lecture Notes in Math. 1317, Springer, Berlin, 1988, 239-249.

[3] G. B. Folland, A Course in Abstract Harmonic Analysis, CRC Press, Boca Raton, FL, 1995.

[4] J. García-Cuerva, K. S. Kazaryan, V. I. Kolyada and J. L. Torrea, The HausdorffYoung inequality with vector-valued coefficients and applications, Russian Math. Surveys 53 (1998), 435-513.

[5] E. Hewitt and K. A. Ross, Abstract Harmonic Analysis, Vol. I, 2nd ed., Grundlehren Math. Wiss. 115, Springer, Berlin, 1979.

[6] A. Hinrichs and H. H. Lee, Duality of Fourier type with respect to locally compact abelian groups, preprint, 2002.

[7] F. Jones, Lebesgue Integration on Euclidean Space, Jones and Bartlett, 1993.

[8] H. König, On the Fourier-coefficients of vector-valued functions, Math. Nachr. 152 (1991), 215-227.

[9] S. Kwapień, Isomorphic characterizations of inner product spaces by orthogonal series with vector-valued coefficients, Studia Math. 44 (1972), 583-595.

[10] J. Peetre, Sur la transformation de Fourier des fonctions à valeurs vectorielles, Rend. Sem. Mat. Univ. Padova 42 (1969), 15-26.

[11] A. Pietsch and J. Wenzel, Orthonormal Systems and Banach Space Geometry, Encyclopedia Math. Appl. 70, Cambridge Univ. Press, Cambridge, 1998.

[12] H. Reiter, Classical Harmonic Analysis and Locally Compact Groups, Oxford Univ. Press, Oxford, 1968. 
[13] W. Rudin, Fourier Analysis on Groups, Interscience Tracts Pure Appl. Math. 12, Interscience Publ., New York, 1962.

Division of Applied Mathematics

Korea Advanced Institute of Science and Technology

373-1 Kuseong-dong, Yuseong-gu

Taejeon 305-701, Republic of Korea

E-mail: ispark@amath.kaist.ac.kr

Current address:

Next generation radio transmission research team

Mobile telecommunication group

Electronics and Telecommunications Research Institute

161 Gajeong-dong, Yuseong-gu

Daejeon 305-350, Republic of Korea

Received June 13, 2002

Revised version December 13, 2004 